\title{
Free Flow of Sweat due to Loss of Surface Tension at Sweat Droplet Causes Water-Induced Skin Wrinkling
}

\author{
Soumya Marasakatla, Karunakar Marasakatla
}

\begin{abstract}
Water immersion skin wrinkling appears to be a result of breaking the balance between the pressure within sweat duct and the pressure exerted by the surface tension of sweat droplet at the pore. When a hand is immersed in water, sweat droplets easily merge within the water causing pressure to drop at the pores. The resulted imbalance in pressure makes the sweat within the duct to flow freely into the water. To prevent loss of water from the body and to maintain homeostasis, the body reacts by restricting blood flow to hand causing vasoconstriction and eventual wrinkling of skin.
\end{abstract}

Keywords: water immersion skin wrinkling, sweat droplet, surface tension, homeostasis, vasoconstriction, Cystic Fibrosis.

(C) 2013 Karunakar Marasakatla

Correspondence to: kmarasakatla@gmail.com

Article is available at: http://vixra.org/abs/1309.0006

Last updated: 09/17/2013 (v3)

\section{Introduction}

Water immersion skin wrinkling (WISW) is a well observed and documented phenomenon. Even though the skin wrinkling is used as a test for sympathetic nerve functioning [1,2,3], the trigger of such a test was eluded for a long period of time. In this article, we investigate theoretical possibilities for the cause of skin wrinkling by taking different physical aspects of the phenomenon into consideration.

Sweating appears to be a physical process involving the pressure within the sweat duct and the surface tension of the sweat droplet at the pore. Sweating surfaces on the skin as a droplet when the pressure within the duct is greater than the pressure exerted by the surface tension of leading edge of sweat. Sweat droplet grows in size until the surface tension of the droplet and the pressure within the sweat duct are in equilibrium. If there is no further increase in the pressure within the sweat duct, no further sweat is formed; unless the sweat droplet is wiped off the skin, or the sweat is evaporated. When the sweat droplet is wiped off the skin, a new droplet starts to form at the pore until equilibrium is reached again.

During the period of intense physical activity, the pressure within the sweat duct increases due to the generation of new sweat at sweat glands. The increased pressure pushes the sweat outward. The droplets grow in size and merge with adjoining droplets to form as drops, eventually sliding off the skin due to increased weight. When the body is in rest, the pressure within the duct and the surface tension of the droplet will be in equilibrium. In these conditions, when a hand is immersed in water, sweat droplets easily merge within the water causing the pressure to drop at the pores. This in turn makes sweat to flow into the water in the absence of substantial counteracting pressure. Sweat glands produce more sweat because of the low pressure within the duct. The loss of water content from the blood, as excessive sweat, continues as long as there is a flow of blood to parts around the sweat glands. To maintain the homeostasis of the body, the nervous system triggers the reduction of blood flow to the palmar region, causing vasoconstriction as reported by Wilder-Smith and Chow [4]. The skin wrinkles due to the loss of volume under the skin.

\section{Discussion}

All the earlier work on skin wrinkling suggests the association of WISW with hyperhidrosis and dilation of 
sweat ducts [5,6,7]. Dilation of sweat ducts suggests a rapid flow of sweat within the ducts. Kabashima et al. [6] reported that skin wrinkling was limited to the iodine-starch positive areas. Further suggesting, as one of the possibility, the skin wrinkling is induced by hyperhidrosis following water exposure. Arkin et al. [7] observed an increase in transepidermal water loss in the form of vapor immediately after skin wrinkling.

During the state of hyperhidrosis, sweat glands continuously generate new sweat causing the pressure to increase within the sweat duct. Excessive pressure within the duct combined with loss of pressure at the pores when immersed in water, can only result in a flow of sweat towards the pores. Dilation of sweat ducts and the transepidermal water loss also suggests the case for flow of sweat towards the pores. The loss of sweat in a resting body triggers the vasoconstriction and eventual wrinkling of the skin. Wrinkles appear on the palmar area only because it has more sweat pores than any other part of the body [8]. More pores cause more sweat, and faster depletion of water from the body; in turn causing faster formation of dense wrinkles.

Many physical aspects of the system influence the speed of sweat flow across the barrier when immersed in water. Surface tension of the sweat droplet along with the pressure within the duct increases with the increase in the density of the sweat. Temperature of the water as reported by Cales and Weber [9] and the tonicity of the water as reported by the Tsai and Kirkham [10] also influences the speed of diffusion of sweat into the water. Another factor that influences the sweat flow is the pressure exerted by the water at the pore. The water column exerts more pressure at deep within the container than at a shallow depth.

In the case of where the leading edge of the sweat is within the duct, the surface tension caused by the minute surface of the sweat is minimal. Therefore the pressure within the duct is also smaller because of the equilibrium between these two forces. In this scenario, when the hand is immersed in water, wrinkles might not form because of the possibility of formation of an air bubble between the sweat and the water. Once the water is diffused into the duct and the bubble is cleared; a water channel will be established between the sweat glands and the water container. At this stage, the palmar area might appear swollen instead of forming wrinkles because of the absorption of water. After establishing a water channel, if the pressure within the duct and the pressure of the water column at the pore are both the same, then wrinkles might not form because of the absence of sweat flow. The flow of sweat might initiate enabling the formation of wrinkles if the hand is exposed to warm water or if the body is subjected to physical activity.

All the previous work showed that wrinkles form on the hand faster when placed under running water, like taking a shower or washing hands under a tap, than placing hands in a bucket full of stationary water of same temperature. Flowing water strips away sweat droplets on the pores as soon as they form. Moreover, the remaining sweat in the duct flows faster into the water flow because of the low pressure created in the flowing water. According to the Bernoulli principle of fluid dynamics, the faster the movement of the fluid, the lower the pressure it creates in the flow. The sweat glands produce more sweat to replenish the loss in the duct. This continuous process of generation of sweat at the glands and merger of sweat into the water flow at the pores, depletes the water content of the blood faster than stationary water. A faster response from the nervous system, and subsequent vasoconstriction, causes the skin to wrinkle faster than the stationary water.

The three primary processes that cools down the body temperature are sweating, respiration through lungs, and radiation from the surface of the skin. If one of the processes are blocked, the other processes needs to increase the rate of heat loss from the body to maintain the homeostasis. In the cases of Cystic Fibrosis, it was observed that the onset of decease causes hyperhidrosis and aquagenic skin wrinkling [7,11]. Possibly, patients with Cystic Fibrosis acquires the characteristics of hyperhidrosis because of the partial blockage of the trachea with mucus. The loss of heat from lungs through exhaling water vapor diminishes due to the blockage of the respiratory tracts. To compensate the reduced cooling through exhaling, the body overloads the sweat glands to produce more sweat to cool down the body temperature. This possibly causes hyperhidrosis, and the related WISW in most of the patients with Cystic Fibrosis. Hyperhidrosis disappears after the cure from Cystic Fibrosis because of the clearing of the respiratory tracts for proper exhaling and the return of normal functioning of the sweat glands.

\section{Conclusion}


Sweating and water immersion skin wrinkling is a dynamic system dependent on many physical factors. It is possible that loss of surface tension of sweat droplet at the pore, when immersed in tap water during hyperhidrosis, might be the primary cause of skin wrinkling. The work presented in this paper coherently explains most of the earlier observations about skin wrinkling. Further study on these lines might shed more light on this interesting phenomenon.

\section{References}

1. Lewis T, Pickering GW. Circulatory changes in fingers in some diseases of nervous system with special reference to digital atrophy of peripheral nerve lesions, Clin Sci 1936; 2:149-175

2. O' Riain S. New and simple test of nerve function in hand, Brit Med J 1973; 3:615-616

3. Bull C, Henry JA. Finger Wrinkling as a test of autonomic function, Brit Med J 1977; 1:551-552

4. Wilder-Smith EP, Chow A. Water-immersion wrinkling is due to vasoconstriction, Muscle \& Nerve 2003; 27:307-311

5. Neri I, Bianchi F, Patrizi A. Transient aquagenic palmar hyperwrinkling: The first instance reported in a young boy, Pediatr Dermatol 2006; 23:39-42

6. Kabashima $\mathrm{K}$ et al. Aberrant aquaporin 5 expression in the sweat gland in aquagenic wrinkling of the palms, J Am Acad Dermatol 2008; 59:S28-S32

7. Arkin $L$ et al. High prevalence of aquagenic wrinkling of the palms in patients with Cystic Fibrosis and association with measurable increases in transepidermal water loss, Pediatr Dermatol 2012; 29:560-566

8. Scheuplein RJ, Blank IH. Permeability of the skin, Physiol Rev 1971; 51:702-747

9. Cales L, Weber R. Effects of water temperature on skin wrinkling, J Hand Surg Am 1997; 22A:747-749

10. Tsai N, Kirkham S. Fingertip skin wrinkling - the effect of varying tonicity, J Hand Surg Br 2005; 30:273-275

11. Elliot RB. Letter: Wrinkling of skin in cystic fibrosis, Lancet 1974; 2:108 